\title{
SÉBASTIEN GALLAND
}

Université Paul-Valéry, Montpellier III

\section{Le poil, le réel et l’informe chez Georges Bataille}

D armi les éléments constitutifs de l'hétérologie ba-

taillienne, le poil a été symptomatiquement occulté par la critique avisée. Pourtant, à examiner la chose de plus près : " Ainsi les guenilles d'Edwarda me regardaient, velues et roses, pleines de vie comme une pieuvre répugnante ${ }^{1}$, le poil est omniprésent dans les récits comme dans les essais de Bataille. Provoquant I'horripilation et la fascination, il participe de l'informe qui marque un rejet de la rationalité et de la philosophie, de la métaphorisation et de l'esthétique, au profit de la laideur, de l'abjection et du bas matériel. Objet hétérogène et irréductible, le poil ne saurait se soumettre au discours, à ses catégories ou ses normes. II borde une zone unheimlich (étrangeté inquiétante) qui confine à l'irreprésentable, et suscite un processus de démétaphorisation où se rencontre le réel sexuel sans nom et sans image. Par là, cet objet irréductible libère la puissance transgressive du sacré, celle de l'érotisme sans doute, mais plus encore celle du dionysiaque qui est volonté de puissance et augmentation du sentiment de vie. Parce que la pilosité atteste de l'énergie vitale commune à l'humain, à l'animal et au végétal, elle est la " part maudite ", l'expression de la grande santé qui, pour Bataille lecteur de Nietzsche, s'inscrit en faux contre les puritanismes anciens ou actuels qui caractérisent les idéaux ascétiques. Déchet de la civilisation, le poil n'en est pas seulement le malaise mais aussi la limite subversive et joyeuse. Chasser, traquer ou raser la pilosité, laquelle

$\overline{1 \text { G. Bataille, Madame E }} d$ warda, Paris, Jean-Jacques Pauvert, 1956, p. 34. 
repousse régulièrement et persiste jusque dans la mort, démontre la force d'un impossible qui ne se laisse pas dominer : le poil comme réel de la vie qui nous déborde, et tisse un lien matériel entre le monde des vivants et le monde des morts. Fétichisée, la pilosité dévoilerait la structure perverse de l'interdit en montrant les limites du déni civilisationnel.

Au commencement était le poil en tant qu'il renvoie à la puissance inépuisable de la nature, une nature productrice et dilapidatrice qui toujours excède les limites et suscite l'inquiétude. La nature se dépense en pure perte, conformément à un principe de vie qui n'advient et ne se déploie que sur le fond de sa propre destruction ${ }^{2}$. Les éléments se consument de manière inconditionnelle et outrancière, et le poil, par ses connotations sexuelles, participe de cette violence frénétique qui veut que la croissance exige la dépense et l'abondance la négation. L'homme n'étant pas un empire dans un empire, la pilosité le rattache immédiatement à la nature. Elle restaure " une intimité perdue " qui arrache l'humain " à la pauvreté des choses ", pour le restituer à "l'économie générale " de l'univers qui tend à l'épuisement libre sans principe de compensation. La division entre "l'économie générale", qui consiste telle la nature " à faire un usage improductif des ressources ", et "l'économie restreinte " soumise à l'utilité sociale et la rationalité calculatrice, passe au cœur de l'individu, et le poil matérialise cette différence irréductible. En effet, le poil est un objet hétérogène qui signale la présence en l'homme d'une vie brute déterminée par l'espèce biologique et l'animalité de la nature humaine que la culture recouvre avec plus ou moins de bonheur.

2 G. Bataille, La part maudite, [dans :] Idem, CEuvres complètes, Paris, Gallimard, 1976, t. 7, p. 31 : «À la surface du globe, pour la m a ti è re vivante en gé néral, l'énergie est toujours en excès, la question est toujours posée en termes de luxe, le choix est limité au mode de dilapidation des richesses $"$. 
Cette vie profonde et muette horripile, elle se fond avec I'horreur de l'ordure, du sexe et de la mort :

Quel est donc essentiellement le sens de notre horreur de la nature ? Ne vouloir dépendre de rien, nous détourner du lieu de la naissance charnelle, nous révolter intimement contre le fait de mourir, généralement nous méfier du corps, c'estàdire, en nousmêmes, de ce qui est accidentel, naturel, périssable, tel apparaît être pour chacun de nous le sens du mouvement qui nous porte à figurer l'homme indépendamment de l'ordure, des fonctions sexuelles et de la mort. ${ }^{3}$

Dans L'impossible, la femme est " belle comme une louve et noire " mais sa nudité "fait peur ", elle est la voie qui mène " à travers les flammes " à l'ordure et la nuit ${ }^{4}$. L'obscénité exaspère l'amour bataillien, le sexe féminin par sa "fourrure " évoque l'animalité, il est assimilé à un " gros rat ${ }^{5}:$ " la nudité n'est que la mort et les plus tendres baisers ont un arrière-goût de rat ${ }^{6}$. Le " secret que le corps abandonne avec la robe " correspond à cette animalité qui conteste la société, ses tabous et ses interdits. Le poil est la trace d'une brutalité originaire et d'une passion exubérante qui perdurent, malgré les tentatives de sublimation de la communauté humaine. Destructeurs des provisions et propagateurs d'épidémies, les rats grouillent dans les bas-fonds. Leurs poils poisseux et crasseux ont partie liée avec ce que la civilisation refoule, et qui touche à l'abject, au dégoûtant et répugnant. Dans sa qualité animale et obscène, le poil excède les structures culturelles qui s'efforcent de le réduire aux règles de la décence. Par là, il est vecteur de division, l'horreur et le dégoût qu'il inspire renforcent l'attrait et la fascination qu'il exerce. La logique homogénéisante du monde rejette l'impropre et l'impur, oubliant que le refoulement et le retour du refoulé sont un seul et même processus. La

${ }^{3} \mathrm{G}$. Bataille, L'histoire de l'érotisme, [dans :] Idem, CEuvres complètes, Paris, Gallimard, 1976, t. 8, p. 79.

${ }^{4} \mathrm{G}$. Bataille, L'impossible, Paris, Minuit, 1962, p. 24 et 32.

5 Ibidem, p. 44-45.

6 Ibidem, p. 100. 
pilosité réintroduit l'immonde dans le monde et, avec elle, c'est le bas matériel qui se rappelle au monde homogène de l'idéalisme. L'hétérogène captive à proportion de l'interdit que I'homogène fait peser sur lui. Pareille tension entre l'homogène et l'hétérogène entraîne un renversement des hiérarchies et des axiologies en matière de jugement moral ou esthétique. La rose n'est plus le symbole de l'amour céleste, sublimé et mystique, ses significations poétiques, virginales et mariales font l'objet d'une démétaphorisation :

les fleurs les plus belles sont déparées au centre par la tache velue des organes sexués. C'est ainsi que l'intérieur d'une rose ne répond nullement à sa beauté extérieure, que si l'on arrache jusqu'au dernier les pétales de la corolle, il ne reste plus qu'une touffe d'aspect sordide. ${ }^{7}$

Loin du " verbiage des vieux poètes ", la fleur " est trahie par sa fragilité ", "la merveilleuse corolle pourrit impudiquement au soleil ": "Puisée à la puanteur du fumier, bien qu'elle ait paru y échapper dans un élan de pureté angélique et lyrique, la fleur semble brusquement recourir à son ordure primitive ${ }^{8}$. L'obscénité de la rose résulte de la conjugaison de la sexualité et de la corruption, du désir et de l'horreur de la pourriture. Démétaphoriser la rose revient à profaner ses significations pour en dévoiler l'envers sordide; ce dernier peut donner lieu à une forme de sacralisation paradoxale, comme dans le cas du culte des reliques. L'interdit investit l'abject d'une dimension sacrée dès l'instant où l'objet exclu est placé en position d'exception, digne d'être révéré à condition de n'être pas touché9.

Le goût de l'abjection dérange l'image du corps policé et trouble l'identité sociale, ce qui libère la puissance

7 G. Bataille, "Le langage des fleurs ", [dans :] idem, CEuvres complètes, Paris, Gallimard, 1970, t. 1, p. 176.

8 Ibidem.

${ }^{9}$ G. Bataille, Les larmes d'Éros, Paris, Jean-Jacques Pauvert, 1971, p. 94 :

" L'interdit religieux écarte en principe un acte défini, mais il peut en même temps donner à ce qu'il écarte une valeur " . 
informe qui s'attache à l'hétérogénéité et plus particulièrement à la pilosité. En tant qu'objet rejeté, le poil fait effraction dans les constructions imaginaires et symboliques pour connoter la présence d'un réel rétif à la représentation. Le réel du corps, du sexe, de la vie et de la mort, est la négation de la figure au profit de l'informe. L'informe pour Bataille prend à revers l'idéalisme, ses théories et ses catégories : il est un réel qui ne se laisse ni saisir ni déterminer ni dominer ${ }^{10}$. L'informe décrit un processus d'érosion de la forme, un délitement, un étiolement, qui renvoie à la précarité des formes face au réel ${ }^{11}$. Parce qu'il ne saurait se laisser clore dans une forme, un bord ou une limite, l'informe marque un rejet de la rationalité, de la philosophie et de l'esthétique; il s'inscrit sur le versant de l'hétérogène et particularise l'abject. L'informe ouvre à la fascination de l'horreur, de l'obscène, du pornographique et du scatologique. II rappelle la prépondérance de l'impur, de la salissure et du déchet. Le poil qui matérialise cet informe est une variante de l'objet a lacanien, cet objet anxiogène qui perturbe la représentation en tant qu'il est l'expulsé de l'image et témoigne d'un reste irréductible à la symbolisation ${ }^{12}$. Le poil est source d'angoisse car il suggère un réel innommable, le fond d'impossible qui constitue l'être de violence et qui est le cri silencieux du corps, de la vie et de la mort. Le poil est un objet séparable du corps, qui trouble son identité imaginaire : cet " abjet » ressortit du corps primitif de la jouissance pulsionnelle, le corps antérieur au stade du miroir et au façonnement culturel. Le poil entache la beauté, et cette tache est le lieu d'émergence de l'angoisse. Dès lors le poil n'est pas sans susciter l'unheimlich, cette inquiétude qui marque le retour du familier sur le mode

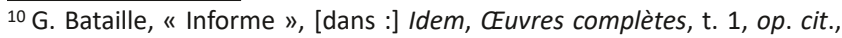
p. 217.

${ }^{11}$ R. Krauss et Y.-A. Dubois, L'informe, mode d'emploi, Paris, Centre Georges Pompidou, 1996, p. 15 et 73.

12 J. Lacan, L'angoisse, Paris, Seuil, 2004, p. 375-389. 
de l'étrangeté : le corps des zones pulsionnelles et des orifices sexuels que l'on préfère dissimuler au regard parce qu'il est synonyme de honte, de laideur, et de souillure ${ }^{13}$. Le poil questionne la frontière du dedans et du dehors. Cet objet révulsif qui pousse sous l'épiderme se rencontre également autour des orifices sexuels qui sont des bords du corps, à la fois intérieurs et extérieurs. L'irruption incongrue du poil étrangéifie le corps spéculaire en laissant apparaître un corps autre, le corps informe dont les limites s'effacent. Le poil qui prolifère sur les pudenda désigne une vie purement organique prélogique, présociale et prépolitique. Le propre du logos étant de se développer dans et par la communauté politique, la logique socialisante exige de raser les poils, alors que le vivant informe et archaïque se signale par une pilosité sauvage et incontrôlable ${ }^{14}$. Le poil partage avec l'informe et l'archaïque d'être un excès qui s'inscrit au-dehors de modèles d'organisation et de classification dont il brouille le fonctionnement et les catégories logiques. II est le symptôme d'un malaise dans la culture, la trace d'un réel impossible à substantialiser parce qu'il se confond avec le mouvement désordonné du vivant, le vestige d'une altérité radicale et opaque qui hante la société des hommes non sans en révéler la vanité. L'humanité sociale peut bien se croire asexuée, elle n'est pas à l'abri de " la saleté des passions " qui est la part obscure, matérielle et asociale de l'humain. Aussi ténu soit-il, le poil est l'organe scandaleux qui confronte I'homme à son inhumanité.

Fût-elle laide et ignoble, la pilosité est une énergie vitale qui est l'être lui-même, l'être qui « est aussi l'excès de l'être " et " montée à l'impossible " hors de toutes limites $^{15}$. Pas plus que le réel, l'être n'est circonscriptible

\footnotetext{
13 Ibidem, p. 90-91.

14 J. Clair, De immundo, Paris, Galilée, 2004, p. 12-14 ; J.-P. Vernant, L'individu, la mort et l'amour, Paris, Gallimard, 1989, p. 193, sur le citoyen spartiate imberbe (joue et menton rasés).

15 G. Bataille, L'érotisme, [dans :] Idem, CEuvres complètes, Paris, Gallimard,
} 
par la pensée et ses concepts, il déborde et se déplace sur un plan horizontal. L'objet poil peut certes provoquer I'horripilation qui dresse les poils sur la peau, il n'empêche qu'il se situe, tout comme le corps intime, dans l'immanence. C'est la nature qui veut que le poil soit pris dans le cycle de la vie et de la mort. La mort est ce que porte toute vie, la reproduction sexuelle consistant à donner la mort en même temps que la vie ; mais la mort confère aussi à l'érotisme sa dimension tragique, la violence de l'étreinte exprimant en creux cette autre violence de mort qui " nous déborde étrangement ". Le " sentiment de gêne à l'égard de l'activité sexuelle " évoque " le sentiment de gêne à l'égard de la mort et des morts ", dont la conscience est apparue avec "l'homme vraisemblablement velu de Néanderthal ${ }^{16}$. La prolifération et la reproduction de l'être ne tirent leur sens que de la mort qu'elles transmettent en dissipant excessivement leur vitalité au moment de l'acte. Aussi Bataille peut-il arguer que la " reproduction et la mort conditionnent le renouveau immortel de la vie; ils conditionnent l'instant toujours nouveau. C'est pourquoi nous ne pouvons avoir de l'enchantement de la vie qu'une vue tragique, mais c'est aussi pourquoi la tragédie est le signe de l'enchantement ${ }^{17}$. Le poil rappelle cette connivence de la vie et de la mort, en tant que sa tige continue de croître jusque sur le cadavre et les tombes. Le narrateur du Bleu du ciel a cette vision brûlante :

Soudain, je pensai à mon rêve : dans un éblouissement, ce que j'avais aimé au cours de ma vie surgissait, comme un cimetière aux tombes blanches sous une lumière lunaire, sous une lumière spectrale : au fond, ce cimetière était un bordel ; le marbre funéraire était vivant, il était poilu par endroits. ${ }^{18}$

1987, t. 10, p. 172.

${ }^{16} \mathrm{G}$. Bataille, Les larmes d'Éros, op. cit., p. 60-61.

${ }_{17} \mathrm{G}$. Bataille, La littérature et le mal, Paris, Gallimard, 1957, p. 20.

18 G. Bataille, Le bleu du ciel, Paris, Jean-Jacques Pauvert, 1957, p. 93. 
La vie, la mort et l'érotisme se conjuguent dans la pierre tombale pareille à un corps sexué. Le poil relie le monde des morts et des vivants, le temps présent et le temps passé. Dans la tradition homérique et présocratique antérieure à l'idéalisme platonicien et ses surgeons judéochrétiens, la pilosité n'est pas la part la plus vile du corps mais le signe de la vigueur et de la virilité : l'incarnation du ménos, cette ardeur vitale qui participe de la jeunesse du monde, dont la mort assure le rejaillissement perpétuel ${ }^{19}$. Dans ce mouvement cyclique de la vie et de la mort se tient la sagesse tragique de Dionysos, le « dieu souffrant et glorifié » dont Nietzsche fut le chantre. D’avoir triomphé de la mort après que les Titans l'eurent démembré, Dionysos est le dieu du renouvellement de la vie. II enseigne l'éternel retour qui est la pensée la plus difficile à assimiler car elle interdit l'idéalisme, élimine le finalisme et le sens acquiescement à une vie sans repos, joie prise à l'existence telle qu'elle est sans la consolation des idéaux rédempteurs ${ }^{20}$. Là où les idéaux dévalorisent la vie au profit d'un arrière-monde intelligible, la pensée de l'éternel retour procède à l'éternisation de la vie notamment sous la forme de la sexualité et de la procréation à l'honneur lors des Dionysies ${ }^{21}$. Pour Nietzsche et Bataille, la sagesse de Dionysos est au service de l'accroissement de cette volonté de puissance qui est augmentation du sentiment de vie. L'homme dionysiaque recherche le terrible " jusqu'à tout luxe de destruction, de dissolution, de négation " afin de se surmonter en dépassant l'idéaliste en lui, l'épreuve dilapidatrice lui permettant aussi de décharger une surabondance vitale dont il pâtit ${ }^{22}$. La sacralité dionysiaque promeut une éthique de la souveraineté de soi sur soi, où

19 J. Clair, Éloge du visible, Paris, Gallimard, 1996, p. 164-165.

${ }^{20} \mathrm{~F}$. Nietzsche, Le gai savoir, $\S 341$, P. Wotling (trad.), Paris, GarnierFlammarion, 1997, p. 279-280.

${ }^{21}$ F. Nietzsche, Crépuscule des idoles, $\S 4$, J.-C. Hemery (trad.), Paris, Gallimard, 1974, p. 148-151 ; G. Bataille, Les larmes d'Éros, op. cit., p. 94-98.

22 F. Nietzsche, Le gai savoir, § 370, op. cit., p. 332-336. 
la limite de la mort non seulement intensifie le désir mais accentue à l'extrême l'élan vital. Dionysos barbu, et son cortège de Satyres et de Silènes velus, appartiennent à un paysage forestier qui se situe au-dehors de la civilisation. Leur sauvagerie traduit la puissance de jaillissement de la nature, une poussée exubérante qui gonfle les végétaux de suc et de sève, et dont participe le poil qui est comme un prolongement de cette humeur vitale (icmas, dynamis) caractéristique de la pousse humaine et végétale ${ }^{23}$. La tige pilaire, la partie émergée du poil formée de la cuticule, de la corticale, et de la moelle, provient de la zone renflée du derme qui se nomme le bulbe à l'instar des plantes.

Aussi la pilosité remplit-elle une fonction subversive propre à l'hétérologie bataillienne. À travers le poil, Bataille condense le pouvoir de fascination qui s'attache à l'animalité des parties honteuses, à leur saleté et leur ordure. Les réactions de dégoût ne sont que le symptôme d'une secrète séduction à l'endroit de ce que l'hygiénisme réprouve. Le poil scandalise aussi fortement qu'il libère des idéaux ascétiques et des morales puritaines. Suivant la ligne du vitalisme nietzschéen, Bataille détecte dans la beauté glabre ou épilée l'indice d'une transformation rancunière de la nature, une négation pessimiste de la vie, du corps et du désir, qui est aussi l'expression de l'impuissance, de la lâcheté et de la tristesse. Michel Leiris qui collabore avec Bataille à la revue critique Documents en 1929 voit dans I'hygiène ancienne ou moderne " une sorte de tabou rationalisé ", au point que la propreté comporte "quelque chose d'essentiellement religieux ${ }^{24}$. Les régions pilaires renferment de mauvaises odeurs qui « attirent les mauvais

${ }^{23}$ H. Jeanmaire, Dionysos, Paris, Payot, 1991, p. 280 ; M. Detienne, L'écriture d'Orphée, Paris, Gallimard, 1989, p. 70.

24 M. Leiris, "Hygiène ", [dans :] G. Bataille et al., Dictionnaire critique, Paris, Prairial, 2016, p. 45. G. Agamben souligne qu'avant la Chute, Adam et Ève portent un "vêtement de grâce " (habit de lumière et de gloire) qui recouvre leur nudité et leur pilosité (G. Agamben, Nudités, M. Rueff (trad.), Paris, Payot\&Rivages, 2012, p. 83-126). 
esprits ", il convient de les nettoyer régulièrement pour s'en prémunir. Et Bataille d'ajouter que la religion est l'ensemble des "prohibitions ", des " obligations " et des " licences partielles " qui " canalisent et régularisent socialement " l'expulsion illimitée des objets impurs rappelant " la dépendance de l'être humain par rapport au donné naturel ${ }^{25}$. Cette " mystique de la propreté » est un avatar de la pensée magico-religieuse, elle définit un rapport au vivant empoisonné de " moraline » qui réapparaît avec les fascismes des années trente. Le corps nazi est représenté glabre, sans couleur ni pilosité pubienne, même s'il peut s'accommoder d'une érotique impudique et fascinatoire ${ }^{26}$. Les idéalismes, les hygiénismes et les fascismes ont ceci de commun qu'ils s'appliquent à détruire I'hétérogène au nom d'une représentation homogène et unitaire du corps matériel27. Le poil qui connote le vivant hétérogène et irréductible n'aura pas droit de cité dans ces systèmes; il sera rejeté tels "les produits d'excrétion du corps humain $" 28$, la "vermine " ou les "parasites" dont la société doit se purifier. De là cette pratique consistant à tondre et raser les déportés à leur arrivée dans les camps de concentration : ceux qui n'étaient plus considérés comme des hommes n'étaient plus non plus des vivants, mais déjà des cadavres expulsés de " l'anus du monde " et promis à une destruction anonyme et industrielle ${ }^{29}$. L'idéalisme, l'hygiénisme et le fascisme sont des cultures de l'interdit qui renforcent la fascination pour la fange et le mal. Leur angélisme génère les conditions de possibilité d'un retour de la bestialité, une bestialité

${ }^{25} \mathrm{G}$. Bataille, "La valeur d'usage de D. A. F. de Sade ", [dans :] Idem, CEuvres complètes, Paris, Gallimard, 1970, t. 2, p. 61, et idem, L'histoire de l'érotisme, op. cit., p. 79.

${ }^{26} \mathrm{~J}$. Chapoutot, Le nazisme et l'antiquité, Paris, PUF, 2012, p. 271-273.

27 G. Bataille, "La structure psychologique du fascisme ", [dans :] Idem, CEuvres complètes, t. 1, op. cit., p. 344-348.

28 Ibidem.

29 J. Clair, Éloge du visible, op. cit., p. 165. 
plus dangereuse encore que la simple animalité, dans la mesure où elle mobilise les instruments d'asservissement de la civilisation :

Les limites donnent à la passion le mouvement crispé qu'elle n'avait pas dans l'animalité : ce mouvement proprement humain [...] mène à des raffinements de jouissance et de cruauté que seuls la civilisation et l'interdit ont rendu possibles par contrecoup. ${ }^{30}$

La transgression étant le propre de l'humain, il apparaît que le mal, la violence et le négatif se nourrissent du respect des valeurs interdites tout comme il n'y aurait pas de négatif, de violence et de mal si l'écart transgressif n'était possible et attirant. L'interdit ne modifie pas la violence humaine mais, " en fondant le milieu humain, il en fait ce que l'animalité ignorait : la transgression de la règle ${ }^{31}$. La société suscite la transgression qui justifie ses interdits et ses tabous, aussi logiquement que la répulsion se transforme toujours de manière plus ou moins latente en attraction et séduction. Cette structure perverse livre la vérité du poil, qui est un fétiche noir dans l'imaginaire bataillien.

Dans son article sur "Le fétichisme ", Freud explique que la " stupeur devant les organes génitaux réels de la femme " entraîne chez le fétichiste la désignation d'un substitut qui prend la place du pénis absent. II s'agit d'une protection contre l'horreur de la castration, qui est la menace de la mort, du réel de la mort ${ }^{32}$. Parmi les fétiches qui conjurent l'affect inquiétant, se trouvent les poils, les cheveux, la fourrure ou le satin, qui fonctionnent telles des synecdoques (la partie pour le tout) de l'organe génital féminin ${ }^{33}$. Le fétiche n'est pas le sexe féminin, il est

$30 \mathrm{G}$. Bataille, "La souveraineté », [dans :] Idem, CEuvres complètes, t. 8, op. cit., p. 47.

${ }^{31} \mathrm{G}$. Bataille, L'histoire de l'érotisme, CEuvres complètes, t. 7, op. cit., p. 47.

$32 \mathrm{~S}$. Freud, "Le fétichisme ", [dans :] Idem, La vie sexuelle, J. Laplanche (trad.), Paris, PUF, 1969, p. 135.

${ }^{33}$ G. Agamben, Stanze, Y. Hersant (trad.), Paris, Payot\&Rivages, 1992, p. 65-69. 
plutôt l'écran derrière lequel se laisse deviner la possibilité de sa présence cachée : ce qui donne sa valeur érotique au poil. Le fétiche est un arrêt sur image, qui fixe le regard à la périphérie du réel traumatique recouvert par l'amnésie infantile. La démétaphorisation, qui transparaît chez Bataille à travers le choix de l'obscène et du sordide, le conduit à privilégier la synecdoque sur la métaphore. Le déni (Verleugnung) concentre le jeu de l'attraction et de la répulsion qui définit le dispositif social et son rapport aux objets expulsés. Le fétichiste s'efforce de faire coexister le déni et la reconnaissance de la castration, son fétiche polarise à la fois des affects de tendresse et d'hostilité ${ }^{34}$. À l'instar des interdits sociaux qui provoquent la transgression, le fétiche est le retour de la représentation refoulée dans le mouvement même du déni. Le réel du sexe est simultanément nié et évoqué par son substitut. Le processus civilisationnel dessine en négatif son contraire : la violence, le désordre et l'impur, qu'il ne peut totalement résorber. Un reste subsiste, un impossible que nul discours ou institution ne saurait atomiser. Les interdits sociaux travaillent plus ou moins à leur insu à pérenniser la passion pour l'obscène et le sordide. La civilisation ne combat pas le mal, elle le produit par et dans ses interdits, elle le repousse tout en le produisant et le produit tout en le repoussant. Le poil se loge en lieu et place de l'inconscient, la part sombre et inavouable de la civilisation occidentale. La pilosité permet donc à Bataille de promouvoir une pensée du bas matérialisme et de l'immanence naturelle incompatible avec les processus de métaphorisation, qui accompagnent les idéalismes hygiénistes ou aseptisants. À l'intellectualisme et ses tentatives de sublimation artistique, morale ou religieuse, il oppose l'excès vital des puissances souterraines qui subvertissent les ordres hiérarchisés et autoritaires. Nul étonnement si Bataille choisit de se reconnaître dans la figure de la « vieille

${ }^{34}$ S. Freud, "Le fétichisme », op. cit., p. 135. 
taupe ", laquelle, au-delà du bestiaire révolutionnaire emprunté à Marx ${ }^{35}$, désigne d'abord un mammifère fouisseur dont le corps est recouvert de poils noirs.

Date de réception de l'article : 13.03.2020.

Date d'acceptation de l'article : 30.04.2020.

${ }^{35} \mathrm{G}$. Bataille, "La "vieille taupe" et le préfixe sur dans les mots surhomme et surréaliste ", [dans :] Idem, CEuvres complètes, t. 2, op. cit., p. 103. 


\section{bibliographie}

Agamben G., Stanze, Y. Hersant (trad.), Paris, Payot\&Rivages, 1992.

Agamben G., Nudités, M. Rueff (trad.), Paris, Payot\&Rivages, 2012.

Bataille G., Madame Edwarda, Paris, Jean-Jacques Pauvert, 1956.

Bataille G., La littérature et le mal, Paris, Gallimard, 1957.

Bataille G., Le bleu du ciel, Paris, Jean-Jacques Pauvert, 1957.

Bataille G., L'impossible, Paris, Minuit, 1962.

Bataille G., Les larmes d'Éros, Paris, Jean-Jacques Pauvert, 1971.

Bataille G., "Le langage des fleurs ", [dans :] Idem, CEuvres complètes, Paris, Gallimard, 1970, t. 1.

Bataille G., "Informe », [dans :] Idem, CEuvres complètes, Paris, Gallimard, 1970, t. 1.

Bataille G., "La structure psychologique du fascisme ", [dans :] Idem, Cuvres complètes, Paris, Gallimard, 1970, t. 1.

Bataille G., "La valeur d'usage de D. A. F. de Sade ", [dans :] Idem, CEuvres complètes, Paris, Gallimard, 1970, t. 2.

Bataille G., " La "vieille taupe" et le préfixe sur dans les mots surhomme et surréaliste ", [dans :] Idem, CEuvres complètes, Paris, Gallimard, 1970, t. 2.

Bataille G., La part maudite, [dans :] Idem, CEuvres complètes, Paris, Gallimard, 1976, t. 7.

Bataille G., L'histoire de l'érotisme, [dans :] Idem, CEuvres complètes, Paris, Gallimard, 1976, t. 8.

Bataille G., "La souveraineté ", [dans :] Idem, CEuvres complètes, Paris, Gallimard, 1976, t. 8.

Bataille G., L'érotisme, [dans :] Idem, CEuvres complètes, Paris, Gallimard, 1987, t. 10.

Chapoutot J., Le nazisme et l'antiquité, Paris, PUF, 2012.

Clair J., Éloge du visible, Paris, Gallimard, 1996.

Clair J., De immundo, Paris, Galilée, 2004.

Detienne M., L'écriture d'Orphée, Paris, Gallimard, 1989.

Freud S., "Le fétichisme ", [dans :] Idem, La vie sexuelle, J. Laplanche (trad.), Paris, PUF, 1969.

Jeanmaire H., Dionysos, Paris, Payot, 1991.

Krauss R. et Dubois Y.-A., L'informe, mode d'emploi, Paris, Centre Georges Pompidou, 1996.

Lacan J., L'angoisse, Paris, Seuil, 2004.

Leiris M., " Hygiène ", [dans :] G. Bataille et al., Dictionnaire critique, Paris, Prairial, 2016.

Nietzsche F., Crépuscule des idoles, § 4, J.-C. Hemery (trad.), Paris, Gallimard, 1974.

Nietzsche F., Le gai savoir, P. Wotling (trad.), Paris, Garnier-Flammarion, 1997.

Vernant J.-P., L'individu, la mort et l'amour, Paris, Gallimard, 1989. 


\section{abstract}

\section{Hair, real and undefined in Georges Bataille}

An emblem of heterology, hair causes horripilation and fascination: it is a part of the shapelessness that signals a rejection of rationality and philosophy, of metaphorisation and aesthetics, favouring instead ugliness, abjectness and material baseness. It verges on an unheimlich (uncanny) zone, which borders on the unrepresentable. Thus, this irreducible object unleashes the transgressive power of the sacred, that, no doubt, of eroticism, but even more so, that of the Dionysiac, which is the will for power and an increase in the feeling of being alive, against ascetic, hygienist or fascist ideals. Hair would be the fetish, attesting to the perverse structure of the forbidden and of civilization.

\section{keywords}

hair, informe, distortion, vitalism, anti-puritanism

\section{mots-clés}

poils, informe, démétaphorisation, vitalisme, antipuritanisme

\section{sébastien galland}

Professeur de culture générale en classes préparatoires à Montpellier. Chargé de cours d'esthétique en Philosophie et en Arts plastiques à l'université Paul-Valéry (Montpellier III). Docteur en Histoire de la Philosophie. Chercheur à l'ENS Lettres et Sciences Humaines à Lyon. Dans ce cadre traduction de la Correspondance de Marsile Ficin. Autres ouvrages : Giordano Bruno, De la triade supérieure; Métaphysique de la lumière ; Marsile Ficin, Choix de lettres; Marsile Ficin, Argument pour la théologie platonicienne; Hildegarde de Bingen et la tradition visionnaire. Articles sur les esthétiques négatives dans l'art et les littératures des XXe-XX|e siècles, ainsi que sur les images de la terreur.

ORCID : https://orcid.org/0000-0002-0217-9417 